\title{
Optimizing dialysis dose in the context of frailty: an exploratory study
}

\author{
Sandra Y. Hernandez-Agudelo ${ }^{1}$ - Carlos G. Musso ${ }^{2,3,6}$ • Henry J. González-Torres ${ }^{2,4} \cdot$ Christian Castro-Hernández $^{1}$. \\ Lina P. Maya-Altamiranda ${ }^{1}$. María V. Quintero-Cruz ${ }^{1}$. Claudio Corradino ${ }^{5}$. Sergio A. Terrasa ${ }^{6}$. \\ Gustavo J. Aroca-Martínez ${ }^{2,7} \cdot$ Andrés Cadena-Bonfanti ${ }^{1,2}$
}

Received: 26 July 2020 / Accepted: 15 December 2020

(c) The Author(s), under exclusive licence to Springer Nature B.V. part of Springer Nature 2021

\begin{abstract}
Introduction Frailty is a multicausal syndrome characterized by a decrease in strength, resistance and physiological function, which makes the individual vulnerable and dependent, and increases his/her mortality. This syndrome is more prevalent among older individuals, and chronic kidney disease patients, particularly those on dialysis. Dialysis dose is currently standardized for hemodialysis (HD) patients regardless of their age and functional status. However, it has been postulated that the dialysis dose required in older patients, especially frail ones, should be lower, since it could increase their degree of frailty. Then, the purpose of this study was to evaluate if there would be a correlation between the dose of Kt/V and the degree of frailty in a population of adult patients on HD.

Materials and methods A cross-sectional study with 82 patients on HD in Barranquilla (Colombia) and Lobos (Argentina) was conducted. Socio-demographic and laboratory data, as well as dialysis doses (Kt/V) were recorded and scales of fragility, physical activity, gait and grip strength were applied. Then these data were correlated by a Spearman's correlation and a logistic regression.

Results CFS, social isolation, physical activity, gait speed, and prehensile strength tests were outside the reference ranges in the studied group. No significant correlation was found between dialysis dose and all the above mentioned functional tests. However, a significant and inverse correlation between physical activity and CFS was documented (score - 1.41 (CI -2.1 to -0.7$)$.

Conclusion No significant correlation was documented between $\mathrm{Kt} / \mathrm{V}$ value and different parameters of the frailty status, but this status correlated significantly and inversely with physical activity in this group. Frailty status in hemodialysis patients was significantly higher in older individuals, although young individuals were not exempt from it.
\end{abstract}

Keywords Frailty $\cdot$ Dialysis dose $\cdot$ Hemodialysis

Carlos G. Musso

carlos.musso@hospitalitaliano.org.ar

1 Departamento de Nefrología, Clínica de la Costa, Barranquilla, Colombia

2 Ageing Biology Unit, Hospital Italiano de Buenos Aires, Buenos Aires, Argentina

3 Facultad Ciencias de la Salud, Universidad Simón Bolivar, Barranquilla, Colombia

4 Doctorado en Ciencias Biomédicas, Universidad del Valle, Cali, Colombia

5 Unidad de Diálisis, Hospital General de Agudos Carlos G. Durand, Buenos Aires, Argentina

6 Research Department, Hospital Italiano de Buenos Aires, Buenos Aires, Argentina

7 Universidad del Norte, Barranquilla, Colombia

\section{Introduction}

Frailty is a multicausal clinical syndrome characterized by a decrease in strength, resistance and body physiological condition, making the individual vulnerable, and increasing his/ her risk of dependence and death. It should be noted the difference between frailty syndrome and comorbidity, as well as between frailty syndrome and disability. Comorbidity is the coexistence of two or more chronic diseases of systemic significance, while disability is the inability to perform at least one of the activities of daily living. However, these conditions can be overlapped and linked, since the burden of comorbidities can lead to a state of frailty, and this in turn, can finally induce disability [1]. 
The importance of detecting the frailty phenotype lies in its close association with a high risk of hospitalization and death [2]. This diagnosis can be established through various tests validated for that purpose, such as a clinical scale based in the degree of autonomy in the performance of daily activities (clinical frailty scale (CFS), the evaluation of the gait speed (gait test) and/or a register of the grip strength of the individual (prehensile strength test) [3].

In chronic conditions frailty phenotype is even more prevalent, as is the case of chronic kidney disease (CKD), a condition characterized by a progressive and irreversible deterioration of renal function due to the reduction of the nephron mass for a period of at least three months [3].

It has been documented that the prevalence of frailty phenotype in adult patients suffering from non-dialysis chronic kidney disease (14\%) is higher than in the general population $(7 \%)$. Likewise, the prevalence of frailty in patients on chronic hemodialysis (HD) is around $42 \%$ (35\% in young people and $50 \%$ in older people) and pre-frailty is $29 \%$, presenting these patients (frailty phenotype) a 2.60 -fold higher risk of mortality and 1.43 fold higher number of hospitalizations, regardless the age, comorbidity and disability [4].

The most validated and universally used parameter to evaluate the dialysis dose is the assessment of the urea removal rate by means of the $\mathrm{Kt} / \mathrm{V}$. In this parameter, "K" is the effective urea clearance (commonly accepted as the solute marker of uremic toxicity), «t» is the time of duration of the dialysis session and $\langle\mathrm{V} »$ is the urea volume of distribution [5]. The $\mathrm{Kt} / \mathrm{V}$ is a crucial determinant in the clinical outcome of the patients on chronic HD, considering as an adequate $\mathrm{Kt} / \mathrm{V}$ value $\geq 1.2$ per session $[6,7]$.

This dialysis dose is standardized for the population on HD regardless of the age and functional status (robust or frail). It has been postulated that the dose of dialysis required in older patients, especially frail patients, would be lower (since the rate of urea production and its volume of distribution are reduced), and that even the conventional HD (three-weekly sessions of 4-5 h) could increase their degree of frailty by induction of hypotension and gait instability [6].

Then, the purpose of this study was to evaluate if there would be a correlation between the dose of $\mathrm{Kt} / \mathrm{V}$ and the degree of frailty in a population of adult patients on chronic HD.

\section{Material and methods}

A cross-sectional study was conducted with 82 adult patients on chronic HD. The subjects evaluated attended two HD centers, one in the city of Barranquilla (Colombia) and the other in the city of Lobos (Argentina), during the period of time comprised between January 2017 and May 2018. Only those who had a HD treatment longer than 3 months, over
18 years of age and who had agreed to participate in the present study, signing the informed consent, were included in the study.

The socio-demographic data of each patient were recorded, as well as some laboratory parameters, hospital stays, and dialysis dose $(\mathrm{Kt} / \mathrm{V})$. The CFS of Rockwood et al. $(2007)[8,9]$ in Spanish was applied to evaluate the patients' biological frailty (Annex, Table A). For evaluating the patients' social frailty, the Social Isolation Score of Pantell et al. (2013) was applied [10] (Annex, Table B). In addition, the Questionnaire of Hierarchical Physical Activity of Reuben et al. (1993) [11] (Annex, Table C), the Gait Speed Test [12] (Annex, Table D), and the Hand-Grip Strength Test [13] (Annex, Table E) were also applied to these patients before their first dialysis session of the study week (Annex).

To evaluate if there was a significant difference in the findings depending of the patient's age, the population was also separated into two groups according to the age: group 1 with young adult patients (18 to 64 years old), and group 2 with older patients ( $\geq 65$ years old).

Then, to determine if there was a significant difference in the evaluated variables among patients with different functional clinical status, these were compared dividing the population in three groups: robust (CFS: 1-3), frail (CFS: 4-5), and very frail (CFS:6-7). It is worth mentioning that there was no terminal patients (CFS: 7-8) in the studied group.

Basic descriptive statistics, the test of statistical significance between two proportions, a Spearman's correlation and a logistic regression were applied to the data. The same evaluation was performed among the different functional groups. The statistical analyzes were performed with the R-CRAN statistical package.

The study was approved by the Institutional Bioethical Committee, and informed consent was obtained from all the participants included in the study.

\section{Results}

A total of 82 adult patients on chronic HD were recruited and divided into two groups: young adult patients (age 18-64 years) and older young patients ( $\geq 65$ years), being the average age $51 \pm 13$ years and $75 \pm 7$, respectively. A predominance of males was found in both groups, $58 \%$ in the young adult patients and $68 \%$ in the older patiens. Regarding the presence of comorbidities, the proportions were similar in both groups, ( $p$ value NS), as was the case of diabetes mellitus type 2, hypertension, and heart failure. Conversely, stroke was significantly more prevalent in the older group (Table 1).

As for the functional markers; the CFS value was higher in both age groups (CFS value $>3$ ) compared to its reference value (CFS $<3$ in healthy people). Additionally, CFS value 
Table 1 Socio-demographic characterization and comorbidities in young and older patients groups

\begin{tabular}{llll}
\hline Socio-demographic & Young adult patients (18-64 years old) & Older patients ( $\geq 65$ years old) & $p$ value \\
\hline No of individuals & 57 & 25 & - \\
Age (years) & $51 \pm 13$ years & $75 \pm 7$ years & $<001$ \\
Gender (\%male) & $58 \%$ & $68 \%$ & NS \\
\hline Comorbidities & Adult (age 18-64 years) & Elderly ( $\geq 65$ years) & NS value \\
\hline DM2 & $42 \%$ & $47 \%$ & NS \\
HP & $85 \%$ & $80 \%$ & NS \\
HF & $9 \%$ & $7 \%$ & $<001$ \\
ST & $0 \%$ & $27 \%$ & \\
\hline
\end{tabular}

$D M 2$ diabetes mellitus type 2, HP hypertension, $H F$ heart failure, $S T$ stroke, $N S$ no significant

Table 2 Evaluation of the different parameters that assess frailty status

\begin{tabular}{lllll}
\hline Evaluation & $\begin{array}{l}\text { Young adult patients } \\
(18-64 \text { years old })\end{array}$ & $\begin{array}{l}\text { Older patients } \\
(\geq 65 \text { years old })\end{array}$ & $p$ value & Reference range \\
\hline Clinical Frailty Scale & $3.8 \pm 1.3$ & $5.5 \pm 5.5$ & $<001$ & $\leq 3$ \\
Social isolation (low score: 4$)$ & $6 \%$ & $13 \%$ & $\mathrm{NS}$ & $\leq 1$ \\
Physical activity (low score: 0$)$ & $55 \%$ & $87 \%$ & 0.05 & $2-3$ \\
Gait speed (m/sec) & $6.1 \pm 3.5$ & $8.3 \pm 2.8$ & 0.03 & $<8$ \\
Hand-grip (kg) & $41.5 \pm 21.6$ & $33.2 \pm 13.7$ & $\mathrm{NS}$ & $57-105$ \\
\hline
\end{tabular}

NS non-significant

was significantly higher in older patients (CFS: $5.5 \pm 5.5$ ) compared to CFS value the young patients (CFS: $3.8 \pm 1.3$ ), $p$ value $<0.01$ (Table 2 ).

With regards the Social Isolation score, it was high (suboptimal) in a low percentage of young adult patients (6\%) and older patients (13\%), and there was no significant difference in this score between the age groups. Regarding the scale of Physical Activity, more than 50\% presented a low score in both studied groups: young adult patients (55\%), and older patients (87\%), being slightly worse this score in the older group $(p$ value $=0.05)($ Table 2$)$.

Regarding Speed Test, the average value was normal in the young adult patient group $6.1 \pm 3.5(\mathrm{~m} / \mathrm{sec})$, while it was abnormal in the older patients group: $8.3 \pm 2.8(\mathrm{~m} / \mathrm{sec})$, being this gait tests significantly worse in the older patients ( $p$ 0.03) (Table 2).

Finally, the hand-grip test was below the reference value in both age groups, and there was no significant difference between the young adult patient and older patient groups $(p=\mathrm{NS})$ (Table 2).

Regarding the serum laboratory parameters and $\mathrm{Kt} / \mathrm{V}$ value, there was no significant differences between both studied groups, except for serum creatinine value which was significantly lower in the older group ( $\left.p:^{<} 0.001\right)$ (Table 3 ).

There was no significant correlation (Spearman) between $\mathrm{Kt} / \mathrm{V}$ value and different parameters of the frailty status, such as the clinical frailty scale, grip strength, gait
Table 3 Serum parameters of the studied patients

\begin{tabular}{lccl}
\hline Parameter & $\begin{array}{c}\text { Young group } \\
(18-64 \text { years })\end{array}$ & $\begin{array}{c}\text { Older group } \\
(\geq 65 \text { years })\end{array}$ & $p$ value \\
\hline Hemoglobin $(\mathrm{g} / \mathrm{l})$ & $10.1 \pm 2.4$ & $10.8 \pm 2.1$ & $\mathrm{NS}$ \\
Hematocrit $(\%)$ & $29.9 \pm 5.1$ & $30.8 \pm 6.5$ & $\mathrm{NS}$ \\
Serum creatinine (mg/dl) & $10.1 \pm 3.3$ & $7.3 \pm 2.7$ & $<0.001$ \\
BUN (mg/dl) & $64 \pm 22.3$ & $57.5 \pm 19.8$ & $\mathrm{NS}$ \\
Serum calcium (mg/dl) & $10.2 \pm 1$ & $8.8 \pm 0.6$ & $\mathrm{NS}$ \\
Serum phosphate $(\mathrm{mg} / \mathrm{dl})$ & $4.8 \pm 1.4$ & $4.4 \pm 1.2$ & $\mathrm{NS}$ \\
Serum albumin $(\mathrm{g} / \mathrm{dl})$ & $3.4 \pm 0.6$ & $3.3 \pm 0.6$ & $\mathrm{NS}$ \\
Kt/V & $1.13 \pm 0.25$ & $1.15 \pm 027$ & $\mathrm{NS}$ \\
\hline
\end{tabular}

$N S$ no significant, $B U N$ blood urea nitrogen

speed and physical activity. Through a logistic regression, when adjusting for the potential confounding effect of the dialysis dose $(\mathrm{Kt} / \mathrm{V})$ and the age, it was found that there was a significant association between CFS and the Physical Activity level, with a score of -1.41 (CI -2.1 to - 0.7) in the CFS. i.e., for each point of increase in frailty, the level of Physical Activity descends 1.4.

Then, the obtained data was reevaluated dividing the studied patients in three groups depending on their functional status: robust $(\mathrm{R})$, frail $(\mathrm{F})$, and very frail (VF). 
Table 4 Socio-demographic characterization and comorbidities in robust, frail and very frail patients groups

\begin{tabular}{|c|c|c|c|c|}
\hline Socio-demographic & Robust & Frail & Very frail & $p$ value \\
\hline No of individuals & 29 & 29 & 24 & - \\
\hline Age (years) & $52 \pm 15$ & $56 \pm 15$ & $68 \pm 13$ & $<0.001$ \\
\hline Gender (\% Male) & $52 \%$ & $66 \%$ & $67 \%$ & NS \\
\hline Comorbidities & Robust & Frail & Very frail & $p$ value \\
\hline $\mathrm{DM} 2$ & $39 \%$ & $38 \%$ & $57 \%$ & NS \\
\hline $\mathrm{HP}$ & $89 \%$ & $94 \%$ & $80 \%$ & NS \\
\hline $\mathrm{HF}$ & $6 \%$ & $13 \%$ & $7 \%$ & NS \\
\hline ST & $0 \%$ & $13 \%$ & $14 \%$ & NS \\
\hline
\end{tabular}

$D M 2$ diabetes mellitus type 2, HP hypertension, $H F$ heart failure, $S T$ stroke, $N S$ no significant

Table 5 Evaluation of frailty parameter among different frailty status

\begin{tabular}{llllll}
\hline Evaluation & Robust & Frail & Very frail & $p$ & Reference range \\
\hline Clinical Frailty Scale & $1-3$ & $4-5$ & $6-7$ & - & $\leq 3$ \\
Social isolation (low score 4) & $17 \%$ & $6 \%$ & $0 \%$ & 0.07 & $\leq 1$ \\
Physical activity (low score 0) & $33 \%$ & $69 \%$ & $100 \%$ & $<0.001$ & $2-3$ \\
Gait speed (m/sec) & $5.4 \pm 2.6$ & $7.7 \pm 2.0$ & $7.5 \pm 5.4$ & NS & $<8$ \\
Hand-grip (kg) & $19.3 \pm 11$ & $20.7 \pm 14$ & $19.1 \pm 16$ & NS & $57-105$ \\
\hline
\end{tabular}

NS non-significant

From this perspective, it was documented that the oldest individuals were in the VF group $(p=<0.001)$, while there was a slightly preponderance of male gender in all the functional groups. Regarding the documented comorbidities, such as diabetes mellitus, hypertension, heart failure, and stroke, there was no significant difference in their prevalence among the three functional groups (Table 4).

Regarding the frailty parameters, there was a significant difference in the percentage of individuals with the lowest social isolation and physical activity scores among the three studied groups: the percentage of individuals with the lowest social isolation score was slightly higher in the $\mathrm{R}$ group ( $p=0.07)$, and the percentage of individuals with the lowest physical activity score was significantly higher in the F and VF groups, particularly in the last one ( $p=<$ 0.001 ) (Table 5). Even though gait-speed and hand-grip tests showed abnormal value in the three studied groups, there was no significant difference among them (Table 5).

Finally, regarding the evaluated serum parameters: hemoglobin, hematocrit, and albumin were abnormally reduced, while creatinine, and BUN were abnormally increased in the three groups. As regards serum calcium and phosphorus, the former showed variable values (low and high) among the studied groups, while the latter showed normal values among them. However, there was no significant difference regarding all the above mentioned serum parameters among the three groups, except for serum hemoglobin, creatinine, and albumin which were significantly lower in the VF group
Table 6 Serum parameters in each frailty group

\begin{tabular}{lclcc}
\hline Parameter & Robust & Frail & Very frail & $p$ value \\
\hline Hemoglobin (g/l) & $11.3 \pm 2.2$ & $10.2 \pm 2.5$ & $9.3 \pm 1.9$ & 0.05 \\
Hematocrit (\%) & $29.9 \pm 6.2$ & $31.2 \pm 4.8$ & $29.2 \pm 5.8$ & $\mathrm{NS}$ \\
Serum creatinine (mg/ & $10.3 \pm 3.4$ & $9.7 \pm 3.0$ & $7.3 \pm 2.8$ & 0.002 \\
$\quad \mathrm{dl})$ & & & & \\
BUN (mg/dl) & $62 \pm 23$ & $63.4 \pm 23$ & $60.7 \pm 19.2$ & $\mathrm{NS}$ \\
Serum calcium (mg/dl) & $8.9 \pm 0.7$ & $8.7 \pm 0.8$ & $12.0 \pm 16$ & $\mathrm{NS}$ \\
Serum phosphate (mg/ & $4.6 \pm 1.4$ & $4.9 \pm 1.4$ & $4.5 \pm 1.2$ & $\mathrm{NS}$ \\
$\quad \mathrm{dl})$ & & & & \\
Serum albumin (g/dl) & $3.5 \pm 0.5$ & $3.6 \pm 0.4$ & $3.1 \pm 0.7$ & 0.025 \\
Kt/V & $1.13 \pm 0.3$ & $1.16 \pm 0.2$ & $1.10 \pm 0.2$ & $\mathrm{NS}$ \\
\hline
\end{tabular}

$N S$ no significant, $B U N$ blood urea nitrogen

(Table 6). Concerning Kt/V value, there was no significant difference in its value among the three functional groups (Table 6).

\section{Discussion}

The majority of patients in both age groups were male. This phenomenon can be explained due to the higher prevalence of male gender among CKD patients [14]. Regarding the similar prevalence of hypertension and diabetes mellitus type 2 documented in both age groups, this finding can be 
explained by the importance of these both conditions as CKD etiology, as well as the high prevalence that hypertension has in CKD patients $[15,16]$. In regards with the higher prevalence of stroke in the older group, it can be explained due to the higher prevalence of this disease in the older individuals, particularly in those suffering from chronic nephropathy [17].

As for the biochemical parameters, the significantly lower serum creatinine in older patients can be attributed to the characteristic sarcopenia of this group, since the magnitude of muscle mass directly impacts on serum creatinine levels [18].

In both age groups, it was documented similar degree of anemia and hypoalbuminemia, being both groups on adequate and similar dialysis (Kt/V) and erythropoietin doses. This phenomenon could reflect a state of malnutrition and/or chronic inflammation (erythropoietin resistance) [16].

With regard to the frailty status, which was evaluated by applying the CFS, gait-speed test, and physical activity score, it was significantly more marked in the older group. Nevertheless, it was found some degree of biological and social frailty in the young group. It should be noted that although there was no significant difference in the grip strength between the groups, it was low for both of them. Moreover, CFS correlated significantly and inversely with physical activity in the studied group, regardless patients' age and dialysis dose. Therefore, these findings reflect the significantly negative influence that CKD have on the deterioration of muscle functionality [19-22].

This is consistent with other literature reports that have documented not only frailty in young CKD patients, but even in children suffering from this condition, reinforcing the concept that frailty should be evaluated even in pediatric population with CKD [20].

Finally, no significant correlation was found between the $\mathrm{Kt} / \mathrm{V}$ value and the frailty markers. Although this could be attributed to the fact that the study did not gather the necessary number of patients to be able to find such correlation, its lack could be interpreted as at that level of $\mathrm{Kt} / \mathrm{V}$ the state of frailty does not depend on the delivered dialysis dose but rather on other variables. In consonance with this finding it is documented that the prognosis of the population on chronic dialysis worsens (higher mortality) when the Kt/V is $<1.1$ [21].

As expected, the oldest individuals and percentage of those individuals with the lowest physical activity scores were in the VF group, since prevalence and degree of frailty progressively increases with aging (Tables 4 and 5) (4). The slightly preponderance of male gender among the three functional groups could be explained due to the higher prevalence of chronic nephropathy in men (7). The fact that there was no significant difference in the prevalence of comorbidities among the three functional groups reinforces the concept that comorbidity and frailty are related clinical scenarios but not identical ones (Table 4) (4).

Regarding the evaluated frailty parameters, the percentage of individuals with the lowest social isolation score was slightly higher in the $\mathrm{R}$ group $(p=0.07)$, perhaps because these patients were able to perform self-care, and consequently had no need of social support network (Table 5). In addition, there was no significant difference in the gaitspeed and hand-grip tests values among the three functional groups, since these tests showed abnormal low values in all of them. This phenomenon could be explained by the sarcopenia and peripheral neuropathy usually present in chronic dialysis patients $(4,7)$ (Table 5).

Regarding the evaluated serum parameters, most of them showed no significant difference among the three studied groups, and their values were the expected for chronic dialysis patients. Serum hemoglobin, creatinine, and albumin were significantly lower in the VF group, phenomenon which could be explained due to the higher inflammatory status (erythropoietin resistance, low serum albumin), and sarcopenia (lower serum creatinine value) which characterized this functional group. Some VF patients also showed hypercalcemia, which could be associated to their immobility syndrome (Table 6). Finally, concerning Kt/V value, there was no significant difference in its value among the studied groups since the same dialysis dose target was sought during renal replacement treatment prescription for every patient.

\section{Conclusion}

In this study, it was found no significant correlation between $\mathrm{Kt} / \mathrm{V}$ value and different parameters of the frailty status, but this status correlated significantly and inversely with physical activity in this group. Frailty status in hemodialysis patients was significantly higher in older individuals, although young individuals were not exempt from it. Finally, very frail dialysis patients showed the highest proportion of patients with low physical activity score.

It is worth pointing out that dialysis dose should be individualized in all patients, but particularly in older and frail individuals since not only standard dialysis dose could be unnecessary high for these patients because dialytic adequacy have been designed based on a series of parameters which are usually significantly modified in these clinical groups, such as patient's total body water, water compartmental distribution, body surface area, and urea generation rate; but also standard dialysis dose could have deleterious effect on these patients by inducing or accelerating frailty status.

Because of that, there is a tremendous need of performing a prospective controlled study, based on these concepts, to 
document, on one hand: which would be an adequate $\mathrm{KtV}$ value in these particular groups, and on the other hand: to explore if dialytic adequacy evaluation should also be based on patient's clinical functional markers, such as clinical frailty scale, hand grip score, and gait rate test, or most likely a combination of them.

\section{Annex}

Table A: Clinical Frailty Scale

1. Very fit $\begin{gathered}\text { People who are robust, active, } \\ \text { energetic and motivated. These } \\ \text { people commonly exercise regu- } \\ \text { larly. They are among the fittest } \\ \text { for their age } \\ \text { People who have no active } \\ \text { disease, symptoms but are } \\ \text { less fit than category 1. Often, } \\ \text { they exercise or are very active } \\ \text { occasionally } \\ \text { People whose medical problems } \\ \text { are well controlled, but are not } \\ \text { regularly active beyond routine } \\ \text { walking }\end{gathered}$
3. Managing well

4. Vulnerable

While not dependent on others for daily help, often symptoms limit activities. A common complaint is being "slowed up", and/or being tired during the day

6. Moderately frail
5. Midly frail

7. Severely frail

8. Very severely frail

9. Terminally ill

These people often have more evident slowing and need help in high orders (finances, medication, transportation, heavy housework)

People need help with all outdoor activities. Indoors they need help with housekeeping, and often have problems with stairs. They also need help with bathing and might need minimal assistance with dressing

Completely dependent for personal care, from either cause (physical or cognitive). Even so, they seem stable and not at high risk of dying

Completely dependent, and approaching the end of life (within 6 months)

Approaching the end of life. This category applies to any people with a life expectancy $<6$ months, who are not otherwise evidently frail

If dementia, the degree of frailty usually corresponds to the degree of dementia
- Mild dementia: includes forgetting the details of a recent events though still remembering the event itself, repeating the same question/story and social withdrawal

- Moderate dementia: recent memory is very impaired, even though they seemingly can remember their past life events well. They can do personal care with prompting

- Severe dementia: they cannot do personal care without help

Table B: Social Isolation Score

- Four type of relationships (1 point for each). Score ranges from 0 (highest isolation) to 4 (lowest isolation).

- Marital status: being married or living together with someone.

- Frequency of contact with other people: having 3 or more interactions with other people per week.

- Participation in religious activities: attending church or religious services 4 or more times per year.

- Participation in other club or organization activities: being member of a club or organization.

Table C: Physical Activity-Reuben Scale

3. Do you participate at least three times a week in a sporting activity or exercise such as swimming, jogging, tennis, cycling, aerobics, gymnastics or other activities, that cause sweating or leave without breathing?

2. Do you walk, at least three times a week, between 9 and 20 blocks $(1.6 \mathrm{~km})$ without rest?

1. Do you walk at least three times a week less than 8 blocks $(0.5 \mathrm{~km})$ without rest?

0 . None of the above.

\section{Table D: Gait speed}

- Seconds it takes you walking four and a half meters at usual pace. The patient is instructed to walk a further meter to avoid slowing near the end point.

- Two measurements are taken and the shortest time (highest speed).

\begin{tabular}{lllll}
\hline Men & & & Women & \\
\cline { 1 - 1 } Height $(\mathrm{cm})$ & Cut point $(\mathrm{seg})$ & & Height $(\mathrm{cm})$ & Cut point $(\mathrm{seg})$ \\
\hline$\leq 173$ & $\geq 7(0.65 \mathrm{~m} / \mathrm{s})$ & & $\leq 159$ & $\geq 7(0.65 \mathrm{~m} / \mathrm{s})$ \\
$>173$ & $\geq 6(0.76 \mathrm{~m} / \mathrm{s})$ & & $>159$ & $\geq 6(0.76 \mathrm{~m} / \mathrm{s})$ \\
\hline
\end{tabular}


Table E: Hand-Grip

- The individual should be seated in a chair with an adduction and neutral rotation shoulder, $90^{\circ}$ flexion elbow, mid prono-supination forearm, and wrist with $0-30^{\circ}$ dorsal flexion and $0-15^{\circ}$ ulnar deviation. The subject is asked to perform the grip with the maximum force by means of a fast but continuous impulse, until reaching the maximum power, registering the measurement of maximum strength reached by the dynamometer needle. The best result of 3 trials of the dominant hand is recorded, with at least $15 \mathrm{~s}$ of recovery between each effort.

- The values given below give guidance on the expected scores for adults according to Fried et al.

\begin{tabular}{|c|c|c|c|}
\hline \multicolumn{2}{|l|}{ Men } & \multicolumn{2}{|c|}{ Women } \\
\hline BMI & Cut point $(\mathrm{kg})$ & $\mathrm{BMI}$ & Cut point $(\mathrm{kg})$ \\
\hline$\leq 24$ & $\leq 29$ & $\leq 23$ & $\leq 17$ \\
\hline $24-26$ & $\leq 30$ & $23-26$ & $\leq 17.3$ \\
\hline $26-28$ & $\leq 30$ & $26-29$ & $\leq 18$ \\
\hline$>28$ & $\leq 32$ & $>29$ & $\leq 21$ \\
\hline
\end{tabular}

\section{Compliance with ethical standards}

Conflict of interest All the authors declare that they have no conflict of interest.

Ethical approval All procedures performed in studies involving human participants were in accordance with the ethical standards of the institutional and/or national research committee and with the 1964 Helsinki declaration and its later amendments or comparable ethical standards.

Informed consent Informed consent was obtained from the patient.

\section{References}

1. Cruz-Jentoft AJ, Baeyens JP, Bauer JM et al (2010) Sarcopenia: European consensus on definition and diagnosis: Report of the European Working Group on Sarcopenia in older people. Age Ageing 39(4):412-423. https://doi.org/10.1093/ageing/afq034

2. Johansen KL, Delgado C, Bao Y, Tamura MK (2013) Frailty and dialysis initiation. Semin Dial 26(6):690-696. https://doi. org/10.1111/sdi.12126

3. Aiello F, Dueñas E, Musso C (2017) Senescent nephropathy: the new renal syndrome. Healthcare 5(4):81. https://doi.org/10.3390/ healthcare 5040081

4. Musso CG, Jauregui JR, Macías Núñez JF (2015) Frailty phenotype and chronic kidney disease: a review of the literature. Int Urol Nephrol 47(11):1801-1807. https://doi.org/10.1007/s11255-015-1112-z

5. Aatif T, Hassani K, Alayoud A et al (2014) Quantification of hemodialysis dose: what Kt/V to choose? Int J Artif Organs 37(1):29-38. https://doi.org/10.5301/ijao.5000236
6. Musso CG, Schwartz P, Deira J, Macias Núñez J (2018) Is it correct to use a unique conventional dialysis prescription in the elderly? Four alternative schedules for dialyzing elderly patients. Int J Ren Dis Ther. https://doi.org/10.31021/ijrdt.20181103

7. Musso CG, Vilas M (2015) Nephroprevention in the very old patient. Rev Colomb Nefrol 2(2):131-136. https://doi.org/10.22265 /acnef.2.2.213

8. García-García FJ, Carcaillon L, Fernandez-Tresguerres J et al (2014) A new operational definition of frailty: the Frailty Trait Scale. J Am Med Dir Assoc 15(5):371.e7-371.e13. https://doi.org/10.1016/j. jamda.2014.01.004

9. Rockwood K, Andrew M, Mitnitski A (2007) A comparison of two approaches to measuring frailty in elderly people. J Gerontol A Biol Sci Med Sci 62(7):738-743

10. Pantell M, Rehkopf D, Jutte D, Syme SL, Balmes J, Adler N (2013) Social isolation: a predictor of mortality comparable to traditional clinical risk factors. Am J Public Health 103(11):2056-2062. https ://doi.org/10.2105/AJPH.2013.301261

11. Reuben DB, Rubenstein LV, Hirsch SH, Hays RD (1992) Value of functional status as a predictor of mortality: results of a prospective study. Am J Med 93(6):663-669. https://doi.org/10.1016/00029343(92)90200-U

12. Montero-Odasso M, Schapira M, Soriano ER et al (2005) Gait velocity as a single predictor of adverse events in healthy seniors aged 75 years and older. J Gerontol A Biol Sci Med Sci 60(10):1304-1309

13. Massy-Westropp NM, Gill TK, Taylor AW, Bohannon RW, Hill CL (2011) Hand grip strength: age and gender stratified normative data in a population-based study. BMC Res Notes 4:127. https://doi. org/10.1186/1756-0500-4-127

14. Huamán CL, Postigo OC, Contreras CC (2016) Características epidemiológicas de los pacientes que inician hemodiálisis crónica en el Hospital Alberto Sabogal Sologuren 2015. Horiz Méd 16:6-12

15. Santamaría-olmo R, Gorostidi-pérez M (2013) Presión arterial y progresión de la enfermedad renal crónica. Nefroplus 5(1):4-11. https://doi.org/10.3265/NefroPlus.pre2013.May.12105

16. Candelaria-Brito JC, Gutiérrez-Gutiérrez C, Bayarre-Vea HD, Acosta-Cruz C, Montes de Oca DM, Labrador-Mazón O (2018) Caracterización de la enfermedad renal crónica en adultos mayores. Rev Colomb Nefrol 5(2):166. https://doi.org/10.22265/acnef.0.0.308

17. Suárez-Escudero JC, Restrepo-Cano SC, Ramírez EP, Bedoya CL, Jiménez I (2011) Clinical, social, occuptional, and of self-perception description of stroke patients. Acta Nerol Colomb 27(2):97-105

18. Portilla Franco ME, Tornero Molina F, Gil GP (2016) La fragilidad en el anciano con enfermedad renal crónica. Nefrología 36(6):609_ 615. https://doi.org/10.1016/j.nefro.2016.03.020

19. Hernández A, Monguí K, Rojas Y (2018) Descripción de la composición corporal, fuerza muscular y actividad física en pacientes con insuficiencia renal crónica en hemodiálisis en una unidad renal en Bogotá. Colombia 11(2):52-56

20. Kehler DS, Ferguson T, Stammers AN et al (2017) Prevalence of frailty in Canadians 18-79 years old in the Canadian Health Measures Survey. BMC Geriatr. https://doi.org/10.1186/s1287 7-017-0423-6

21. Tattersall J, Farrington K, Gentile G et al (2018) Is Kt/V useful in elderly dialysis patients? Pro and Con arguments. Nephrol Dial Transplant 33(5):742-750. https://doi.org/10.1093/ndt/gfy042

22. Capotondo M, Musso CG (2019) Nephroprevention in the elderly. In: Musso CG, Jauregui J, Macías-Núñez JF, Covic A (eds) Clinical Nephrogeriatrics. Springer, Cham, pp 133-146

Publisher's Note Springer Nature remains neutral with regard to jurisdictional claims in published maps and institutional affiliations. 